\title{
El autoconocimiento como condición para construir una personalidad moral y autónoma
}

\author{
Montserrat Payá
}

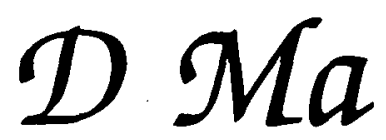

A continuación, se analizan las relaciones entre autoconocimiento $y$ desarrollo moral. Se consideran tres ámbitos temáticos que posibilitan el concocimiento de uno mismo: autodescubrimiento, autoposición ante la realidad interpersonal y autoposición ante la realidad macro-social. Se formula una propuesta de actividades dirigidas en especial a los alumnos del primer ciclo de la Educación Secundaria. También se ofrecen prototipos de actividades para otros tramos educativos.

\section{INTRODUCCION}

Dentro de los objetivos finales de la educación, el autoconocimiento cobra una significación especial, caracterizado bien sea como construcción de la propia identidad, elaboración del proyecto de vida personal, autoconcepto positivo, etc. Igualmente ocurre en el ámbito del desarrollo moral. El autoconocimiento adquiere una dimensión esencial, ya que constituye un punto de partida en la realización de todos aquellos procesos necesarios para construir una personalidad moral, autónoma y responsable. Así, no se puede plantear la reflexión en torno a conflictos de valor, si, primero, no se es consciente de la propia opinión. Tampoco se pueden potenciar, adquirir o modificar determinadas actitudes vitales, si, con anterioridad, no ha habido una autoobservación y una valoración de lo que se desea y de lo que no.

El razonamiento moral, la actitud crítica ante el entorno macrosocial, la relación interpersonal... necesitan de la proyección del conocimiento de sí. Pero, además, éste puede ser también un recurso para construir conocimiento sociomoral. Siendo conscientes de nuestros sentimientos, opiniones y de las razones que los guían, nos encontramos en mejores condiciones para entender los de 
los demás, sin que esta comprensión implique aceptación pasiva, sino más bien el inicio de un proceso de diálogo. Se trata, pues, de una tarea constante y dinámica.

\section{AUTOCONOCIMIENTO Y DESARROLLO}

El autoconocimiento, más que una técnica de educación moral, constituye una finalidad a alcanzar. Su significado, excesivamente amplio e impreciso en ocasiones, excede el carácter procedimental para devenir objetivo, aunque la interacción entre ambos dificulta enormemente su delimitación. Asimismo, se encuentra íntimamente vinculado a otras técnicas de educación moral. Por una parte, es un paso previo y necesario para llevar a cabo el proceso de clarificación de valores. De hecho, este último sería su continuación y consecuencia: «cuando se reconoce el propio yo, se forjan los valores» (Raths, L. E.; Harmin, M.; Simon, S. B. (1967), pág. 214). Por otra parte, el conocimiento de sí implica la aplicación de habilidades de autoobservación y autoevaluación ${ }^{1}-$ las cuales, a su vez, inciden sobre aquél incrementándolo-, que originarán, probablemente, la puesta en práctica de actividades de autorregulación.

Podríamos definir el autoconocimiento como «el esfuerzo por saber lo que sentimos, lo que deseamos, lo que nos impulsa a actuar y creemos necesitar, lo que pensamos y las razones que tenemos, los valores que aceptamos y defendemos y, en definitiva, quiénes somos ante nuestros propios ojos» (Puig, J. M. ${ }^{a}$ (G.R.E.M.) (1991), pág. 3). Asimismo, este esfuerzo o tarea para el conocimiento de sí tiene un carácter diacrónico: incorpora las dimensiones proyectiva futuro- y biográfica - pasado-, posibilitando de esta forma la construcción y consecución del propio proyecto de vida.

Se podría estructurar el autoconocimiento en tres dimensiones: la propia identidad, la autoestima ${ }^{2}$ y la autoconstrucción. Transversalmente a estos tres ámbitos, se hallarían la toma de conciencia de los procesos anteriores y la autoconfianza. Estos cinco aspectos se interrelacionan constantemente. Así, el autoconocimiento debe continuarse en una autoestima objetiva y positiva. Ello constituye el marco necesario para iniciar la construcción de aquellos valores, actitudes, formas de ser, etc., que cada persona desee poseer. La toma de conciencia, tanto del contenido o conocimiento en sí como de los procesos que se llevan a cabo, se va produciendo de forma simultánea — si bien, no automáticamente-, y como consecuencia de todo ello, poco a poco, se genera confianza en uno mismo, al combinarse la aceptación de la propia identidad junto a una creencia y disposición activa de cambio o transformación.

De forma aproximativa, se pueden determinar tres grandes áreas temáticas para, a partir de ellas, motivar y posibilitar el conocimiento de sí: el autodescubrimiento, la autoposición ante la relación interpersonal y la autoposición ante la realidad macrosocial. Por autodescubrimiento entendemos la expresión y toma de conciencia de los propios sentimientos, gustos, temores, preocupaciones, deseos, etc., en definitiva, cómo somos y qué queremos. La segunda área, la autoposición ante la relación interpersonal, pretende el análisis de la propia postura en la relación con el grupo de iguales, familia y todas aquellas personas que son próximas y relevantes, en definitiva, cómo nos relacionamos y cómo tendría que ser. Finalmente, la autoposición ante la realidad macrosocial pretende poner al descubierto los propios principios, las controversias entre valores, 
etc., que, muy probablemente, se plasmarán en opiniones, actitudes y acciones concretas ante la problemática social que se someta a consideración, es decir, qué pensamos sobre... y qué podemos hacer ai respecto. La reflexión en torno a estas tres áreas posibilitaría la consecución de las dimensiones citadas en el párrafo anterior.

Finalmente, queremos dedicar unas líneas a poner de relieve la relación, ya esbozada, entre autoconocimiento y una correcta autoestima. Es por demás conocida la importancia que ésta tiene para la propia motivación y conducta, así como su fuerza para conseguir objetivos o metas propuestas y resolver conflictos. Un autoconcepto positivo redunda en mayor esfuerzo y perseverancia en aquella tarea que la persona se ha determinado, siendo por lo tanto más probable que la consiga y que tenga mayores posibilidades de desarrollo. Por el contrario, una autoestima negativa implica que la persona dedique, de entrada, menos esfuerzo y constancia a la tarea propuesta, lo cual, a su vez, redunda en menor probabilidad de éxito y, por lo tanto, de gratificación y desarrollo. Muchas veces es más determinante la creencia en las propias posibilidades que la posesión misma de las capacidades, medios o soluciones para conseguir tal o cual cosa. Se puede decir que el autoconcepto determina la conducta, el pensamiento y las reacciones emocionales ante situaciones conflictivas, $y$, por consiguiente, que influye en la relación interpersonal (BANDURA, A. (1987) pág. 418). Mediante un trabajo sistemático y continuado sobre el autoconocimiento, se pueden asentar las bases e incidir, de forma positiva, sobre la autoestima.

\section{UNA PROPUESTA DE ACTIVIDAD}

Dado que se ha de considerar el autoconocimiento en su doble carácter - como finalidad u objetivo y como medio o técnica-, es necesario determinar, con precisión y en primer lugar, qué área de contenido se pretende trabajar y graduar las actividades en función de aquélla. Esta afirmación, fundamental -y, también, obvia - para iniciar todo proceso educativo en cualquier área de conocimiento, cobra especial relevancia en este ámbito en el que la falta de claridad y sistematización puede conducir fácilmente a confusión e, incluso, insatisfacción con la actividad desarrollada. Así, en la propuesta ${ }^{3}$ que seguidamente presentaremos, la temática se centró en los conceptos de autoconocimiento y de heteroconocimiento, presentándolo como un posible instrumento para la relación con los demás. De esta forma, se estaba trabajando tanto el autodescubrimiento como también la autoposición en la relación interpersonal.

El programa que se diseñó incluía una primera aproximación - a partir de un texto que ponía de manifiesto la necesidad, importancia y, en ocasiones, dificultad que entrañaba dedicar un tiempo a pensar sobre sí mismo. A este respecto, se proponía un ejercicio de autoobservación sobre la distribución del propio tiempo y, como consecuencia de éste, otro de autorregulación para poder dedicarse también, en equilibrio con las obligaciones, a lo que realmente se deseaba. Asimismo, mediante la literatura, se mostraba también la dimensión biográfica del autoconocimiento, la evocación de acontecimientos que, por uno u otro motivo, estaban presentes en el propio recuerdo. Las actividades sucesivas intentaban facilitar la reflexión y toma de conciencia de la propia identidad desde la perspectiva del autodescubrimiento y de la relación interpersonal. Una de ellas es la que aquí proponemos: 
Adivina quién...

\section{Aquí jugaremos a las adivinanazas.}

Escribe en un papel, sin poner tu nombre, la respuesta - muy breve y concretaa estas cuestiones:

a) Cómo eres.

b) Qué te gusta.

c) Qué es lo más importante para ti.

Ahora, excoged uno e intentad adivinar a quien corresponde.

a) ¿Qué opinas de este juego?

b) ¿Habéis sabido muchas veces de quién se trataba o, por el contrario, os ha resultado difícil?

c) ¿Cuál crees que puede ser el motivo?

La finalidad de esta actividad era que los alumnos y alumnas sintetizaran aspectos significativos de su personalidad, conocieran mejor a los demás y se percataran de la diferencia entre conocer y creer conocer. En su aplicación, se había de especificar al grupo que la primera pregunta no hacía referencia a cuestiones físicas, sino de carácter y forma de ser. Por otra parte, también se les pedía, al adivinar la persona de quién se trataba, que justificaran con motivos su elección. Al finalizar la actividad, se les preguntaba si habían descubierto aspectos nuevos o, incluso, insospechados en los compañeros/as. Ello constituía una forma interesante y amena de darse a conocer y de conocer a los demás. Además, la implicación y la participación estaban casi aseguradas y había muchos aspectos que incidían de forma positiva en la autoestima de los alumnos/as. Obviamente, no es un ejercicio que destaque por la cantidad, cualidad y profundidad en lo que a autoconocimiento se refiere, pero consideramos que es, por lo menos, una forma válida para comenzar.

\section{ORIENTACIONES PRACTICAS}

Para elaborar un programa sobre autoconocimiento, es conveniente compaginar dos aspectos en la determinación del tema. Por una parte, resulta muy adecuado partir de los intereses del grupo-clase, ya que, de esta forma, se aseguran la participación e implicación. Pero, además, se deben plantear también aquellos otros aspectos que cada educador/a considere necesarios trabajar en su clase. Por ejemplo, en los primeros cursos de la Primaria, puede resultar muy motivador comentar los propios gustos y aficiones, pero, paralelamente, nos podemos proponer también que sean conscientes tanto de los propios gustos como de la diversidad dentro del grupo, y de que esta diversidad es beneficiosa para todos. A grandes rasgos y por lo que se refiere a autodescubrimiento, se puede sugerir comenzar, en los primeros cursos, por los aspectos de tipo físico, capacidades y limitaciones, los ya citados gustos y aficiones, etc., para ir ampliando hacia los rasgos de personalidad y carácter. Igualmente, la relación interpersonal se puede enfocar en base a situaciones concretas entre iguales y con la familia que sirvan para descubrir el propio rol, para utilizar este conocimiento en posibles situaciones conflictivas y de desacuerdo, etc. Incluso se pueden trabajar en los primeros cursos temas macrosociales, siempre que haya una vinculación directa con la experiencia del grupo, como ocurre, por ejemplo, con la ecología.

Paralelamente, se ha de procurar facilitar actividades para la autorregulación, que supongan que la alumna o el alumno se planteen un objetivo concreto 
a conseguir y determinen los pasos para alcanzarlo, y también para la autoestima, poniendo de manifiesto las potencialidades y cualidades de cada persona reforzándolas, aceptando las propias limitaciones, etc. Igualmente, es muy aconsejable, en íntima relación con las actividades, verbalizar y conceptualizar todo el proceso, ya que de esta forma se facilita la toma de conciencia. En función de la edad, se necesitará una mayor o menor explicitación por parte del educador/a. Así, en los primeros cursos, será muy conveniente verbalizar tanto el proceso (interrogación, reflexión, expresión, etc.) como la información resultante. En edades más avanzadas, dicha toma de conciencia se puede plantear ya como conceptualización y valoración crítica de los procesos realizados.

Para ello, es fundamental que el clima del aula y de la escuela en general se caracterice por una atmósfera de confianza y de respeto a la diferencia y a la pluralidad, donde se puedan entablar libremente procesos expresivos y comunicativos. De hecho, la tarea de autoconocimiento se ha de concebir como un objetivo que no se consigue tan sólo con la aplicación de actividades a este propósito, sino que necesita de este clima general —que tendría que ser extensivo al ámbito familiar-, y, especialmente, de la valoración e interés personal. Precisamente, este factor, la interiorización, será el que facilitará la transferencia a múltiples situaciones, problemas, controversias de valores, etc. que adoptan dimensión real con la construcción paulatina y progresiva de la propia personalidad moral y del proyecto de vida. A este respecto, las habilidades para la autodirección surgen como un recurso imprescindible y como una continuación totalmente necesaria y natural del conocimiento de sí mismo.

Los ejercicios sobre autoconocimiento presentan una tipología muy variada. Además de poder utilizar la técnica de clarificación de valores, se puede recurrir a procedimientos como el diario personal o la escritura autobiográfica a propósito de acontecimientos especiales por uno $\mathrm{u}$ otro motivo ${ }^{4}$. Igualmente, se pueden dedicar varias sesiones para que cada alumno/a explique o escriba acerca de lo que sabe hacer muy bien, o en torno a experiencias positivas... También se puede elaborar un cuestionario sobre sentimientos, opiniones, etc. respecto a una serie de temas directamente relacionados con los intereses del grupo y pasarlo varias veces a lo largo del tiempo, observando posibles cambios, así como preparar unos guiones de entrevista para conocer a los otros. En realidad, se trata de un ámbito muy flexible donde la creatividad de cada educador/a y las necesidades de cada grupo en concreto resultan esenciales tanto en el diseño de actividades como también en la elaboración del programa.

Pasamos, finalmente, a presentar dos actividades que han sido elaboradas para el ciclo educativo 14-16 años (segundo nivel de la educación secundaria obligatoria). La primera ejemplifica la dimensión biográfica y proyectiva del autoconocimiento a través de la propuesta de diario personal y de la conceptualización de dichas dimensiones. La segunda se caracteriza por introducir la dimensión de autoconstrucción como una tarea a realizar desde la realidad cotidiana:

\section{Diario de un adolescente}

Escribir sobre uno mismo, sobre lo que nos ocurre, lo que sentimos, pensamos, hacemos..., es decir, escribir sobre la propia vida puede ser tambień una buena manera de trabajar para la autoconstrucción personal. A esta escritura autónoma y libre sobre la propia vida es a lo que llamamos autobiografia. Hay diversas maneras de realizar autobiografías, una de ellas puede ser a través de la redacción de diarios personales. Estos podrian definirse como aquellos relatos en los que se intenta hacer una reflexión escrita 
en torno a lo que nos ba ocumido a lo largo de un día. Es también una manera de expresar los sentimientos, reacciones y estados de ánimo que hemos tenido delante de situaciones o momentos determinados.

En definitiva, es una manera de explicarnos a nosostros mismos cómo somos y cómo actuamos, el por qué de nuestras reacciones, con el fin de ayudarnos a conocernos y también a dirigir y reenfocar nuestra manera de pensar y de hacer. Quizás si os mostramos un ejemplo de un diario lo veréis con más claridad.

«Hacía tiempo que no abría este diario, pero la verdad es que últimamente me han ocurrido tantas cosas que ni tan sólo he tenido tiempo para escribir. Es curioso, a veces me pregunto por qué escribo o para quién escribo. De hecho si escribo es porque siento esa necesidad, no sé como explicarlo, necesito sacar todo lo que llevo dentro, hablarme a mí misma, porque de hecho si escribo lo hago para mí misma, no creo que esto lo llegue a leer nunca nadie. Ya me estoy liando, como siempre. ¿Por qué seré tan complicada? Debo hacer un esfuerzo por no romperme tanto el coco. Cuanto más pienso más me embrollo. A veces pienso que pienso demasiado. Bueno, pensar un poco ya va bien, pero quizás me hago una montaña de las cosas, ¿no? Ahora mismo, con todo lo de la marcha de papá quizás esté demasiado preocupada. De hecho ya hace un año y medio que se fue de casa, y yo ya lo tendría que haber asumido, al menos esto es lo que me decían. Recuerdo el día que los padres me dijeron que habían decidido separarse y que papá se iba de casa... Yo hubiera deseado morirme. Los amigos me decían que no me preocupara, que con el tiempo lo iría asumiendo, pero no sé qué me ocurre que cuanto más tiempo pasa peor me siento. De todas formas, tampoco creo que todo sean historias mías. Mamá cada día está más irritable y no me deja vivir en paz, todo el día controlándome. Que si ¿a dónde vas? ¿a qué horas llegas?, y sobre todo estudia, estudia... Ya estoy harta de sus absurdos proteccionismos. ¿Qué piensa, que soy una cría de diez años? Pues sé perfectamente lo que hago. De acuerdo que soy un poco apasionada y doy la imagen de ser alocada, pero yo no soy así. A mí me gusta divertirme, pero nunca me he pasado de la raya.

Con papá era muy diferente, por lo menos él me entendía más y me dejaba salir por las noches. Ahora, en cambio, parece que esté en una prisión. ¡Qué palo! Y encima sólo me ha faltado ponerme a salir con Toni. Mamá se ha puesto histérica, parece que lo haga expresamente para amargarme la existencia. Pero ¿que no comprende que salir con Toni es lo único maravilloso que me ha ocurrido desde que papá se fue? ¡Y suerte que tengo a Toni! Él me escucha y nos lo pasamos bien juntos, pero, claro, a mamá no le gusta nada que vaya vestido de esa forma. Creo que tendré que tomar alguna decisión, porque yo no puedo continuar viviendo de esta forma. Sí, creo que lo mejor será que me fije algunos objetivos para intentar mejorar esta situación, y en caso de que no mejorara, pues ya veríamos. Primero hablaré con mamá y le expresaré todo lo que pienso y siento. Todavía no lo he hecho nunca, pero creo que ya va siendo hora. Procuraré comprenderla, porque ella también lo debe estar pasando mal, pero le debe quedar muy claro que hemos de hacer un esfuerzo, por parte de las dos, para mejorar nuestra relación. Después hablaré con papá. Ya sé que no se ha vuelto a ver con mamá, pero todavía se llaman por teléfono para arreglar algunos asuntos que tienen pendientes. Quizás papá le pueda hacer entender que debe confiar más en mí. También le propondré a papá, si a mamá no le parece mal, que quedemos más frecuentemente para ir a comer o a cenar. Quiero también mantenerlo como un amigo y quizás esto me haga sentir mejor. Finalmente tendré que convencer a mamá de que Toni, aunque vista de esa manera, es un buen chico y a mí me ayuda mucho.

¡Uf!, estoy cansada... Ya continuaré otro día».

1.-Subraya frases o párrafos del texto que más te hayan interesado y explica por qué.

2.- ¿Cuales serían las características de la escritura autobiográfica? Es decir: ¿De qué se habla en el texto? ¿Cómo se habla? ¿A quién va dirigido...?

3.- ¿Para qué piensas que le ha podido servir a la protagonista?

4.-¿ ¿Has escrito alguna vez un diario? ¿Por qué sí o por qué no?

5.- ¿Consideras que escribir sobre la propia vida te puede ayudar en algún aspecto? ¿En cuál?

6.- ¿Crees que la protagonista de este diario es libre? ¿En qué aspectos sí y en cuáles no? 
Escribir sobre la propia vida, sobre lo que se bace, piensa, siente, etc. nos ayuda también a elaborar el pensamiento sobre nuestra persona, en definitiva a conocernos mejor y a saber cuál es la trayectoria a seguir en nuestra vida.

Te proponemos abora que intentes escribir un diario de tu vida, poniendo especial atención en aquellos aspectos positivos y negativos que te han marcado de una manera especial. Es decir, cuáles son las mejores y peores cosas que te han pasado últimamente, cómo te ban afectado y en qué, cómo te ban becho sentir, etc...

- Para comenzar escribe dos páginas poniendo un título y justificándolo.

${ }_{-}$¿Sobre qué otros temas autobiográficos te gustaría escribir?

\section{Nuestro futuro}

En la autoconstrucción personal intervienen tres dimensiones temporales diferentes. Nuestro pasado, todo aquello que bemos vivido y bemos becho, nuestra bistoria, que nos ba marcado de manera particular y ba ido configurando una manera de ser y bacer en el presente. Un presente sobre el que tenemos un poder de intervención real que, a pesar de estar condicionado por nuestro pasado, podemos ir modificando, renovando, cambiando o manteniendo. Y, además, todo lo que somos, lo somos también en perspectivas de un futuro. Hemos de pensar que lo que bacemos en nuestro presente condicionará también, en cierto modo, nuestro futuro y por lo tanto tenemos siempre la posibilidad de dirigir y guiar nuestro quehacer bacia aquello que realmente querriamos y deseariamos ser.

Asi pues, podemos escribir y reflexionar sobre nuestro pasado, sobre nuestro presente $y$ también sobre aquello que querríamos y deseariamos ser $y$ bacer en un futuro. Este último aspecto será muy importante para poder autodirigir la vida en función del objetivo que tengamos.

- Por esto queremos proponerte que hagas un autorretrato de tu futuro. Sueña e intenta tu vida a los treinta años. ¿Cómo te gustaría ser? ¿Qué te gustaría hacer? ¿Qué te gustaría pensar? ¿Qué tipo de vida te gustaría llevar?... Escribelo y después, si te parece bien, coméntalo y compáralo con tus compañeros.

\section{Notas}

${ }^{1}$ En: Bandura, A. (1987). pp. 446-448, se puede encontrar más información acerca de la influencia de las habilidades de autoevaluación sobre el autoconocimiento.

${ }^{2}$ Cabe distinguir, con Bandura (1987), la autoestima de la autoeficacia, si bien ambas «contribuyen, cada una por su parte, a la calidad de vida» (pág. 435). Desde este planteamiento, la autoestima tiene un fuerte componente social y cultural que no se da en la autoeficacia, la cual se limita a la valoración de las capacidades «per se».

${ }^{3}$ Aunque en un principio estaba elaborada para 7. ${ }^{\circ}$ de E.G.B., se aplicó también con idénticos resultados en $8 .^{\circ}$.

${ }^{4}$ En los primeros cursos, se puede substituir la escritura por el dibujo o la expresión oral.

\section{Referencias}

AA.VV. (1992). «Los contenidos». Cuademos de Pedagogía. (Tema del mes: Educación moral) n. ${ }^{\circ}$ 201. Marzo, 1992, pp. 15-18.

Bandura, A. (1987). Pensamiento y acción. Barcelona: Martínez Roca. Biblioteca de Psicología, Psiquiatría y salud. Serie Universidad.

Curwin, R. L. L. y Curwin, G. (1984). Cómo fomentar los valores individuales. Barcelona: CEAC. Col. Aula práctica.

Puig, J. M. a (G.R.E.M.) (1990). Conocimiento y trabajo sobre sí mismo. Documento mecanografiado no publicado. Facultad de Pedagogía. Universidad de Barcelona.

(1991). Diseño curricular. Primer nivel de concreción. Documento mecanografiado no publicado. Facultad de Pedagogía. Universidad de Barcelona.

Raths, L. E.; Harmin, M.; Simon, S. B. (1976). El sentido de los valores y la enseñanza. México: Unión Tipográfica Hispano Americana. 
El autoconocimiento como condición para construir una personalidad moral y autonóma Montserrat Payá Sánchez

$C L \& E, 1992,15$, pp. $69-76$

Resumen: El conocimiento de sí se caracteriza, especialmente, por su carácter transversal. Así, se puede afirmar que constituye más un objetivo a alcanzar que una simple estrategia o técnica dentro del curriculum de educación ético-moral. En este artículo, además, se presenta en relación con el desarrollo de una adecuada autoestima, la influencia en la interacción social, y la construcción de la propia personalidad moral.

Datos sobre el autor: Montserrat Payá Sánchez es licenciada en Pedagogía y miembro del Grupo de Investigación en Educación Moral (G.R.E.M.) de la Universidad de Barcelona. Desde 1989 imparte clases de Etica - niveles 7. y 8. de E.G.B.- en diversos colegios públicos de Cornellá del Llobregat (Barcelona).

Dirección: Departamento de Teoría e Historia de la Educación, Facultad de Pedagogía, Universidad de Barcelona, Baldiri Reixac, s/n 08028 Barcelona. Tel (93) 3333466.

(C) De todos los artículos deberá solicitarse por escrito autorización de CL\&E y de los autores para el uso en forma facsímil, fotocopia o cualquier otro medio de reproducción impresa. CL\&E se reserva el derecho de interponer las acciones legales necesarias en aquellos casos en que se contravenga la ley de derechos de autor. 